\title{
SONOGRAPHIC EVALUATION OF FOCAL AND DIFFUSE HEPATIC LESIONS
}

Hima Bindu P1 ${ }^{1}$ Anil Kumar Shukla², N. Krishnappa ${ }^{3}$

\section{HOW TO CITE THIS ARTICLE:}

Hima Bindu P, Anil Kumar Shukla, N. Krishnappa. "Sonographic Evaluation of Focal and Diffuse Hepatic Lesions". Journal of Evolution of Medical and Dental Sciences 2014; Vol. 3, Issue 45, September 18; Page: 11114-11129, DOI: $10.14260 /$ jemds/2014/3451

ABSTRACT: AIM: To study ultrasonography as prime imaging modality for evaluation of focal and diffuse liver lesions and its confirmation by fine needle aspiration. METHODOLOGY: Patients presenting with liver diseases were included in the study. Ultrasonography was done as initial imaging method, and then guided Fine Needle Aspiration was done to correlate our findings. RESULTS: Ultrasonography was highly sensitive and specific in diagnosing liver abscess, malignant liver tissue \& metastasis. For non-malignant liver lesions like haemangioma, cystic and hydatid lesions, fatty liver and cirrhosis showed sensitivity up to $80 \%$, and specificity up to $90 \%$. CONCLUSION: Above data suggests that ultrasonography has high sensitivity and specificity for overall diagnosis and it helps in guiding Fine Needle Aspiration for confirmation of diagnosis.

KEYWORDS: Ultrasonography, Fine Needle Aspiration, Focal and diffuse hepatic lesions.

INTRODUCTION: Liver diseases like infections, metabolic and neoplastic are of the most significant causes of morbidity and mortality. Clinical assessment is difficult and unreliable however Ultrasonography (USG) has been an accepted method for diagnosis of focal \& diffuses hepatic lesions because of its rapidity in performance \& high sensitivity. ${ }^{1}$ It is cheaper, easily available, effective, quick, painless, can be repeated, non-invasive with no radiation hazard. USG gives valuable information regarding site, size, number and nature of lesions with their relation to surrounding structures. With development of high frequency transducer, it has found important applications for imaging liver, spleen, kidneys and other abdominal organs. ${ }^{2}$

Focal hepatic lesions are frequently missed with one modality, and then detected with another. ${ }^{3}$ USG is used as first line imaging investigation in patients with jaundice, right upper quadrant pain and hepatomegaly 4 . It's preferred to assess patients considered for resection of primary or metastatic liver tumors. Due to its ability to image in any oblique plane it can localize lesions to an anatomic segment or sub segment of the liver. In addition, it can be used as imaging guide for FNAC (Fine Needle Aspiration Cytology) or therapeutic drainage of abscesses.

The guided FNAC of the liver is a safe, simple, cost-effective and accurate method for cytological diagnosis of hepatic lesions like diffuse, focal, nodular and cystic lesions with good sensitivity and specificity. ${ }^{5}$ This study has been conducted on 118 patients to diagnose hepatic lesions by USG and to assess the validity in relation to FNAC diagnosis.

MATRERIAL \& METHODS: A prospective study of 118 cases of focal and diffuse hepatic liver lesions diagnosed by ultrasonography in the Department of Radiodiagnosis. All these cases which were found to have liver lesions were subjected to FNAC for confirmation of finding and establishment of final diagnosis. Inclusion criteria - (a) All patients presenting with varied clinical features suggestive of liver diseases like - Right upper quadrant pain or mass, pyrexia of unknown origin, jaundice, 
constitutional symptoms including malaise, loss of weight. (b) Abnormal liver function tests. (c) Congenital disease involving liver. (d) All patients of either primary tumour or metastasis.

Exclusion criteria - (a) Patients with history of blunt abdominal trauma. (b) Patients without USG features suggestive of liver disease. (c) Granulomatous diseases of liver. Equipment - In the present study, gray scale real time sonographic examination was carried out using $3.5 \mathrm{MHz}$ curvilinear transducer at our hospital, by Voluson 730 Pro machine.

Patient preparation and scanning technique - Prior to USG informed consent, detailed history and brief clinical examination done. Majority of patients were kept nil by mouth for few hours and in some no prior preparation required. Acoustic jelly was thoroughly applied over the skin to act as a coupling agent. Liver was scanned in various planes like sagittal, parasagittal, transverse, oblique, subcostal, intercostal and coronal planes. Comprehensive scanning of other upper abdominal organs was also done.

Various USG features of hepatic liver lesions were observed which include - Number of lesions - single or multiple. Location - Lobar (right, left or both lobes) and segmental distribution. Echogenicity - Hyper, hypo, anechoic or mixed. Size, shape and margins - Exact size, shape like round, oval or irregular, margins poorly or well defined, regular or irregular. Overall assessment of liver size, portal and hepatic veins involvement, biliary tract and gall bladder, lymphadenopathy, aorta and its branches and ascites. FNAC of these detected focal and diffuse hepatic lesions was done.

It was avoided initially in those patients with prolonged bleeding, clotting or pro thrombin time and decreased platelet counts. After correction of these abnormalities, patients were subjected to FNAC. It involves two steps - (1) Accurate USG localization of lesion - It was located with its borders marked on skin by marker and optimal puncture site at center of marked area. The distance between lesion and skin surface was measured.

(2) Aspiration equipment - 22 gauge needle, sterile gloves, sponges, saline and local anesthetic (2\% xylocaine) if needed. Preparation - Patient was advised to fast overnight to minimize gas occurring over areas of interest and to prevent lung aspiration in cases of adverse reaction. Emergency drugs and blood transfusion facilities were kept ready. Technique - Skin was carefully scrubbed and field was draped in sterile fashion.

Needle with stylet was inserted and firmly plunged up to desired depth. Stylet was then removed and $20 \mathrm{cc}$ syringes was attached and aspirated material was smeared on slides. Tip of needle was confirmed to be present within lesion by USG. After the procedure punctured site was washed and simple adhesive bandage was placed. The cells were stained and examined by cytopathologist. Fluid material obtained from cysts was centrifuged $2500 \mathrm{rpm}$ for 15 minutes and sediments stained and examined.

For diagnosis of hydatid lesion, special precaution was taken; it was done in intensive care unit, so as to take action immediately if at all any hypersensitivity reaction occurs. Before procedure hydrocortisone and antihistamine was given. Statistical tests applied were - Sensitivity, specificity, positive predictive value, negative predictive value, chi - square test and cross tab procedure (contingency coefficient).

RESULTS: We have done a detailed analysis of USG \& FNAC findings and came across various observations. We have seen 118 cases in all up to 60 years of age. In 31-40 years range, 25\% cases and between 31 to 50 years of age maximum i.e. $56 \%$ cases and below 10 years only $4 \%$ cases were 
seen. In our study $73 \%$ were males and $45 \%$ were females. Males had increased predilection for liver diseases (Table -1).

Primary malignant tumors, metastasis, abscess have highest incidence in the age group of 4150 years with 13\%,11\% and 8\% cases respectively. Metastatic deposits not found below 20 years, however heamangioma, simple and hydatid cysts were seen up to the age of 60 years. Fatty liver and cirrhosis has highest incidence in the age group of 31-40 years with 5 \& 3\% cases respectively. Diffuse hepatocellular carcinoma have highest incidence in the age group of 51- 60 years with $2 \%$ cases.

Males were predominantly affected by focal and diffuse hepatic lesions than females. In liver abscess M: F was 1.6:1 (22:13), in malignant tumors and metastasis ratio was 1.2:1 (15:2) \& 1.2:1 (12:10) respectively. Mean age incidence of focal lesions among males and females was 41.06 and 42.5 years and of diffuse lesions it was 41.23 and 43.8 years respectively.

Clinical symptoms like pain, fever, hepatomegaly, jaundice \& tenderness were the main. Out of 98 cases, 53\% with pain, 29\% had fever and only 31\% had hepatomegaly clinically and $7 \%$ presented with jaundice (Table -2). In study group 58\% had solitary and $40 \%$ cases had multiple lesions. About lobar involvement 63 cases (64.28\%) right and 15 cases (15.1\%) on left side with both lobes in 20 cases $(20.62 \%)$.

USG features of liver tumor varied in all of 27 cases, majority i.e. 14 (51.8\%) hyperechoic, 4(14.8\%) hypoechoic and 9 i.e. (33.4\%) were mixed echogenic. Out of these 17 (62.9\%) were solitary and 10 (37\%) showing multiple lesions of HCC (Figure-1). USG features of 22 cases of metastasis, majority 8 were hypoechoic, 4 hyperechoic, 5 showed bull's eye lesion and rest showed mixed echogenic features.

USG features of 35 cases of liver abscess, majority i.e. 24\% were hypoechoic, 7\% anechoic and $4 \%$ hyperechoic. Out of these 20 (57.2\%) were solitary and 15 (62.8\%) multiple. Right lobar involvement was noted in 22 (62.8\%), left in $8(22.8 \%)$ and both in $5(14 \%)$ cases. By USG we saw 98 cases of focal lesions of these $27 \%$ were primary, 22\% metastases, $5 \%$ were heamangiomas, $35 \%$ liver abscesses, $4 \%$ cysts and remaining $5 \%$ as hydatids (Figure-2).

These cases were referred to department of pathology, for the confirmation of diagnosis by FNAC. Results indicated 33 cases of abscess, 26 of primary, 22 metastases, and remaining diagnosed by FNAC were 6 haemangioma, 5 hydatids and 6 cystic lesions. Chi- square test - Pearson chi-squareValue is 168.8. Df is $25 . \mathrm{p}<0.001$, very highly significant.

Contingency coefficient test value $=0.836, \mathrm{p}<0.001$. Out of 27 cases which were diagnosed as primary malignant tumors, 22 were confirmed by FNAC and remaining 5 were false positive on ultrasound. Of the 35 cases of liver abscess, 31were confirmed, 2 cases were false negatively diagnosed on ultrasound were confirmed by FNAC.

By USG 22 cases were diagnosed as metastases, of which 17 were confirmed by FNAC, remaining 5 were false positive. By USG 5 cases were diagnosed as heamangioma, of which 3 were confirmed by FNAC and 2 were false positive. Cystic lesions were 4 of which 3 were confirmed by FNAC with 1 case as false positive. Hydatid cyst was seen in 5, of which 4 were confirmed by FNAC and 1 was false positive.

There is significant association between USG finding and FNAC findings (Chi square $\left(\mathrm{X}^{2}\right)=$ 168.8, $\mathrm{p}<0.001$.Contingency Coefficient value of $0.851 \mathrm{p}<0.001$ was found to be highly significant. Statistical values of the study - USG was highly sensitive and specific in malignant liver tumor with 
sensitivity of $84.6 \%$ and specificity of $93 \%$. For metastasis it showed sensitivity of $77.2 \%$ and specificity of $93.4 \%$. Liver abscess has sensitivity of $93.9 \%$ and specificity of $93.8 \%$.

Diagnosis of hemangioma, cystic and hydatid lesions showed specificity of $97.8 \%, 98.9 \%$ \& 98.9\% respectively (Table-3). Out of the total 20 cases studied by ultrasound as cirrhosis, 2 were hepatitis and remaining 3 cases were diffuse hepatocellular. FNAC was done on all the 20 cases and the results indicated 10 of fatty liver, 6 as cirrhosis, 2 as hepatitis and the remaining 2 as diffuse hepatocellular carcinoma (Table - 4, 5, 6).

(Chi square) $\mathrm{x}^{2}=21.32$ at 9 Df. $\mathrm{P}<0.01$, highly significant, Contingency coefficient $=0.81, \mathrm{p}<$ 0.001. By USG 9 cases seen as fatty liver, 8 were confirmed by FNAC. Two cases were false negative, diagnosed as hepatitis and diffuse hepatocellular carcinoma which were diagnosed by FNAC as fatty liver.

USG diagnosed 6 cases as cirrhosis, 5 were confirmed by FNAC. 1 was false positive on ultrasound and was proved as hepatocellular carcinoma by FNAC. Two cases of hepatitis by ultrasound, 1 were confirmed by FNAC and 1 was false positive. One was confirmed as cirrhotic nodule on FNAC. Of the 3 cases of hepatocellular carcinoma were diagnosed by ultrasound 1 was confirmed as hepatocellular carcinoma, 2 were false positive.

There is significant association between USG findings and FNAC findings (Chi-square value $=$ $21.32 \mathrm{p}<0.001)$. Even contingency coefficient value of 0.836 was found to be significant $(\mathrm{p}<0.001)$. Statistical value of the study of diffuse cases diagnosing fatty liver \& cirrhosis ultrasound showed sensitivity of $80 \%, 83.3 \%$, and specificity of $90 \%, 92.8 \%$. In diagnosis of hepatitis and diffuse hepatocellular carcinoma showed specificity of $94.4 \%, 88.89 \%$ (Table-7).

DISCUSSION: Ultrasound was first utilized for nautical purposes after the sinking of the Titanic in 1912. This 'echolocation' technology was used to detect icebergs, which later expanded during WW I and WW II to identify submarines. The first medical ultrasound was used in 1942. USG have been widely used in the diagnosis of liver disease in the past 20 years. It has a big advantage of being realtime which means if a swept image can be repeated and displayed frequently enough then a moving picture is produced in the same way as cine photography.

This study comprises of USG evaluation of focal and diffuse hepatic lesions with FNAC correlation. The FNAC is taken as gold standard in comparing and coordinating the diagnosis made by USG. The various focal and diffuse hepatic lesions are discussed as follow - (A) Focal Hepatic Lesions Following lesions are commonly seen.

CYSTS: USG should be considered the imaging modality of choice; it can accurately localize primary cysts within the liver. The most common single location is the lower edge of the right lobe of liver. By USG they are uni locular anechoic and can be single or multiple (Figure-3). Polycystic Liver Disease Is an embryological mal-development associated with polycystic disease of kidney ( $50 \%$ of patients). Sonographic features consist of innumerable contiguous watery cysts of 2 to $3 \mathrm{~mm}$ in diameter. Peripheral cysts distort the liver surface \& multiple may distort the normal architecture considerably. Acoustic enhancement may produce an impression of abnormal pattern in addition to the cysts.6,7

PRIMARY MALIGNANT LIVER TUMORS: In our study they are second most common lesions encountered. USG pattern of these were studied. Other important findings included presence of 
ascites, indentation and compression of vessels (hepatic and portal vein) and thrombosis of portal vein (Figure-7, 8).

Hepatocellular Carcinoma (HCC) -It is the most common primary hepatic tumor. Viral-related cirrhosis is a leading risk factor for this. ${ }^{8}$ Macro nodular (post necrotic) type of cirrhosis, with a frequency of $13 \%$ to $24 \%$ and with the micro nodular of hemochromatosis $7 \%$ to $22 \%$. It is rare in children. USG is sensitive and integral part of screening for hepatocellular carcinoma in high risk countries like India.

Patterns of tumor growth in HCC - (a) Nodular - most common, consists of multiple, variable sized nodules up to $5 \mathrm{~cm}$ in diameter. Each nodule has an arterial branch supplying it. (b) Massive may have one or several small satellite nodules. (c) Diffuse infiltrative- tiny indistinct nodules and its appearance are hard to differentiate grossly from cirrhosis. The major blood supply to the tumor is the hepatic artery. Portal vein invasion is present grossly in $25 \%$ to $40 \%$ of cases and hepatic vein invasion and even the growth into the right atrium can occur (Figure-4).

USG appearance can be divided into four major categories - (a) Echo free. (b) Echogenic. (c) Mixed \& (d) diffuse infiltration. Color doppler imaging may demonstrate a tangle of vessels within the tumor, indicating hypervascularity, arterio-venous shunting or both. Small tumors may appear diffusely hyperechoic. The highly reflective pattern is most frequent, being present in about half of all cases. Vascular invasion is common and should suggest the diagnosis. ${ }^{9}$

The sensitivity of FNAC is very high, varying between $91 \%$ and $95 \%$ with a specificity of $92 \%$ $100 \%{ }^{10,11}$ USG guided FNAC of the liver plays more role in diagnosis and classification of liver disease than ultrasound alone, as it requires greater precision to reach diagnostic accuracy. ${ }^{12}$

METASTASIS: About half of the patients presented with hepatomegaly or jaundice, another half of patients no clinical features. Out of this $2 / 3$ were multiple and $1 / 3$ cases were solitary lesions. High incidence of multiple was due to vascular blood flow pattern. Majority of metastasis to liver in our study were from gastrointestinal primary spread by hematogeneous route.

Liver is one of the commonest sites for metastatic spread which may occur through the portal vein, hepatic artery, lymphatics or by direct extension from tumor in adjacent organs. It may be found at any position in the liver and are usually multiple. A characteristic feature of multiple liver metastases is the narrow range of size (Figure-5).

\section{ULTRASOUND APPEARANCES:}

a) Focal lesions are commonest and pattern is of echo poor masses. These are recognized by distal enhancement caused by them. They may contain clear fluid but contain debris when the fluid represents tumor necrosis. This type tends to have shaggy walls and is less likely to be mistaken for simple cysts. ${ }^{13}$

b) High1y reflective lesions may be surrounded by an echo-poor band which may be fine or a few $\mathrm{mm}$ thick. This is called "Bull's eye" pattern and is more often seen in larger lesions. Highly reflective and target lesions are typically of tumors originating in the gastrointestinal tract and urogenital tract.

c) Calcified lesions have very intense echoes and may show shadowing if they are sufficiently large. Calcification commonly occurs in secondaries from colorectal and gastric carcinomas as well as neuroblastomas. ${ }^{14}$ Hypervascular lesions are less common than hypovascular metastatic 
lesions. Tumors in this group include metastasis from renal cell carcinoma, islet cell carcinoma, carcinoid, malignant pheochromocytoma and thyroid carcinoma. ${ }^{15}$

d) The different types of metastatic lesions are adenocarcinoma $86 \%$, squamous cell carcinoma $2 \%$, while the round cell neoplasm, ovarian, renal \& small cell carcinoma lung each are $2.3 \%$.

HEMANGIOMAS: They are the most common benign tumors of the liver. Unless very large, they are asymptomatic and do not disturb liver biochemistry. Clinically life threatening haemorrhage has been reported but its rare. ${ }^{16}$

Two forms occur - (a) Cavernous type where large sinusoidal blood vessels are present and (b) Capillary type where numerous small vessels occur. Cavernous is the most common benign tumour of the liver, can occur at any age and is most prevalent in the posterior segment of right lobe. Most lesions are smaller than $5 \mathrm{~cm}$ in size. The spectrum of appearance on ultrasound is variable (Figure-6).

However the majority have a very distinctive pattern. This is of a sharply defined homogeneously hyperechoic round tumor without an hypoechoic peripheral border. ${ }^{17}$ They are bright due to multiple interfaces between the walls of cavernous sinuses and the blood contained within. A minority of hemangioma may present as an isochoric or hypoechoic mass relative to the liver parenchyma. Hemangiomas larger than $2.5 \mathrm{~cm}$ are reported to show posterior acoustic enhancement.

Some hemangiomas, especially if they are large (more than $5-6 \mathrm{~cm}$ in diameter) present a heterogeneous ultrasound echo pattern. The anatomical basis for this is thought to be due to thrombosis, fibrosis, degeneration or hemorrhagic necrosis. Majority of lesions are solitary than multiple. Those lesions with size $<2 \mathrm{~cm}$ were hyperechoic, $2-5 \mathrm{~cm}$ were predominantly echogenic and $>5 \mathrm{~cm}$ showed a mixed echogenic pattern. ${ }^{18}$

LIVER ABSCESS: In the present study number of cases was seen. They are mainly of two types - (a) Pyogenic \& (b) Amoebic.

a) PYOGENIC: It is usually a complication of an intra-abdominal infection with direct portal venous spread to liver. Common etiological sites include biliary tract, appendiceal abscess, crohn's disease and colonic diveirticulitis. ${ }^{19}$ E.coli is the commonest pathogen but both aerobic and anaerobic organisms are frequently found. Ultrasound will usually show a spherical, oval or slightly irregular echopoor lesion with distal enhancement. This pattern is present in $75 \%$ of cases. A significant number of abscesses can be higher in reflectivity than the adjacent normal liver. This may be related to air or micro bubbles within fluid or to a mixture of differing contents producing strong interface. Large amount of gas will produce the typical appearances associated with parenchymal gas. In chronic abscess a wall of variable thickness may be present. They are located usually at the centre and rarely touching hepatic capsules. Many abscesses can be conservatively treated without drainage procedure and hence appropriate patient selection for surgical or percutaneous drainage is done.

Purulent material aspirated from a focal lesion should always be subjected to microbiological investigations. Routine smears should also be prepared and screened for neoplastic cells, as tumor metastasis for example from a glandular primary in bowel or from squamous cell carcinoma can 
undergo necrotising degeneration, simulating an abscess. Amoebic abscess shows abundant necrotic cells with fewer inflammatory cells.20

Pyogenic abscess demonstrates a marked neutrophilic infiltrate with debris. ${ }^{21,22}$ Clinical presentation is in the form of abdominal pain, swinging fever, anorexia and sweating. Single abscesses tend to be gradual in onset and cryptogenic, whereas multiple abscesses present with acute systemic features.

Ultrasonography is the preferred initial method of imaging for liver abscess as it is noninvasive, cost effective and can be used to guide aspiration to identify the causative organisms. ${ }^{23}$ Pyogenic abscesses are multiple, amoebic are solitary. Amoebic abscess are larger in size than pyogenic. Three ultrasound aspects are found- Hypoechoic is the most frequent, followed by heterogenous \& anechogenic. ${ }^{24}$

b) AMOEBIC LIVER ABSCESS: Worldwide they are more common than pyogenic ${ }^{25}$. It's the most common, non-enteric complication of amoebiasis and occurs in an average of $10 \%$ of patients having clinical amoebic infection. It is endemic throughout Asia and Africa, usually occurring after gastro-intestinal infection with entamoeba histolytica, but hepatic amoebiasis can occur coincident with colonic diseases. World's $10 \%$ of population is chronically infected and is the third commonest parasitic cause of death. ${ }^{25}$ There is strong tendency for male preponderance and 3rd to 4 th decade is the vulnerable age group but any age can be affected (Figure-7,8).

Ultrasonography has ability to rapidly detect hepatic abscess at different stages. It shows round or oval shaped hypo echoic lesion with well-defined margin ${ }^{26}$. They are usually peripherally situated touching the liver capsule (subcapsular). ${ }^{27}$ Lesions are primarily hypoechoic compared to normal parenchyma, almost always shows distal acoustic enhancement, a feature that is less consistent with pyogenic abscess. Whenever an abscess is discovered by ultrasound, should be followed by aspiration. After aspiration some strong reflective areas may be present. Untreated abscess may undergo calcification with consequent acoustic shadowing. Some may show bizarre sonographic appearance like irregular echogenicity, interspersed with disorganized low level echoes. In such cases there may be difficulty in making a diagnosis based on ultrasound features alone. USG can be used as a guide for aspiration in order to identify the causative organisms of liver abscess. ${ }^{28}$

HYDATID LESION: Ultrasonography is the most commonly used imaging technique with high diagnostic accuracy rate. In our study we saw 5 male cases, 1 was solitary lesion in right lobe, 1 was multiple in right lobe and the other multiple lesions in both lobes of liver. USG showed 2 hyperechoic lesions with multiple daughter cysts giving 'spoke wheel' appearance.

One case was hypoechoic with typical 'water lily' sign and other 2 were anechoic. Hydatid disease of the liver is parasitic infestation caused by the species of cystodes-echinococcus granulosus and is endemic in our country. Liver is the most frequently involved organ where more than $50 \%$ of cysts found. Cysts are multiple in $40 \%$ of cases and about in $25 \%$ of patients with liver disease also have lung cysts.

Parent cyst develops in the liver, after the ingested larva gains entry in to portal venous system by penetrating the intestinal mucosa. Smaller daughter cysts arise from the germinal epithelium which is the endocyst. Cyst size may vary from 1 to $20 \mathrm{~cm}$, in endemic areas all liver cysts 
are considered to be hydatid unless proven otherwise. The cyst appears as a well-defined sonolucent mass with smooth borders and good posterior enhancement.

The presence of complete rim of calcification suggests as inactive lesion. Debris, consisting of sand and scolices may be present within the lesion. It may also be possible to discrene the two layers of the wall of hydatid lesion. Detachment of inner germinal layer from the exocyst produces 'Water Lilly' sign. The collapsed germinal layer is seen as an undulating linear collection of echoes either floating in the cyst or lying in the most dependent portion.

The free floating blood capsules and scolices form white sediment known as "hydatid sand".

The development of daughter cysts from the lining germinal layer produces a characteristic appearance of "cyst within a cyst". This appearance is extensively characteristic, producing 'cartwheel' or 'honey comb' appearance (Figure-9). Another pattern is 'Rosette' pattern, which is also characteristic of a hydatid with daughter cysts.

Capsule is well outlined but the inner architecture show circular array of cysts and a solid centre. ${ }^{29}$ Aspirated fluid from hydatid cyst is turbid and thick, fragments of the hyaline laminated cyst wall membrane are readily demonstrated. The diagnosis is confirmed by the demonstration of scolices or refractile hooklets. ${ }^{30}$ Aspirate from hydatid cyst yielded thick, turbid fluid which showed refractile dagger-shaped hook lets and fragments of laminated membrane along with degenerating hepatocytes and mixed inflammatory cells. ${ }^{31}$

Ultrasonography showed a sensitivity of $92.3 \%$ and specificity of $98.3 \%$ in diagnosing these lesions.32 It is found that the diagnosis is certain when one can image daughter cysts. But other sonographic appearances cause confusion in diagnosis and may be mistaken for hepatic abscess, necrotic tumor, simple cyst or cystadenoma of liver.

DIAGNOSTIC VALIDITY TEST RESULTS OF VARIOUS FOCAL LIVER LESIONS: For above hemangiornas, cystic and hydatid lesions, sensitivity, specificity, positive and negative predictive values were calculated. Definitive diagnostic precision could not be attributed due to less number of cases enrolled in the study. However, USG with its typical features can diagnose above lesions with high diagnostic accuracy, obviating needle confirmation in majority of cases.

(B) Diffuse hepatic lesions - (a) Fatty Infiltration. (b) Cirrhosis.

(a) FATTY INFILTRATION: constitutes majority encountered by us. Sonographic appearance of fatty liver varied depending on amount of fat. Moderate increase in hepatic echogenicity, slightly impaired visualization of diaphragm and intrahepatic vessels was noted in majority. Fatty infiltration is an acquired disorder of metabolism resulting in accumulation of triglycerides within hepatocytes. Sonography may be varied depending on amount of fat and whether deposits are diffuse or focal.

Diffuse steatosis may be - (1) Minimal diffuse increase in hepatic echogenicity, normal visualization of diaphragm and intrahepatic vessels. (2) Moderate increase in hepatic echogenicity, slightly impaired visualization of diaphragm and intrahepatic vessels. (3) Severe - marked increase in hepatic echogenicity, poor penetration of posterior segment of right lobe of liver, poor or no visualization of diaphragm and intrahepatic vessels. ${ }^{33}$

Severe fatty infiltration often results in an enlarged liver with diffuse increased echogenicity. Acoustic penetration may be decreased, resulting in indistinctness of blood vessels and the diaphragm. The normal echogenicity of the liver is determined by comparing the liver echogenicity with that of the cortex of the kidney. ${ }^{34}$ Hepatic steatosis is the most common finding, which favored 
the cytological diagnosis of fatty liver. It generally involves the entire liver but may produce a circumscribed, nodular lesion as focal fatty change. 31

(b) CIRRHOSIS: Cirrhosis is second most common lesion encountered constituting 6 cases (30\%). About half of the patients presented with hepatomegaly and abdominal distension. Majority visualized as coarse echo texture with nodular surface of liver. Three cases were diagnosed as diffuse hepatocellular carcinoma. One case presented with clinical features of right upper quadrant pain, tenderness, hepatomegaly and fever. It is the diffuse process characterized by fibrosis and conversion of normal liver architecture into structurally abnormal nodules.

There are 3 major pathologic mechanisms which in combination create cirrhosis - cell death, fibrosis and regeneration. USG findings include changes in the shape of the liver, parenchymal inhomogeneity and nodularity of the liver, notably at the surface (Figure-10). Surface nodularity may be the only sonographic sign of cirrhosis. There is relative enlargement of caudate lobe and fibrotic shrinkage of right lobe. ${ }^{34}$

USG visualized as coarse echotexture, nodular surface, regenerating nodules as isoechoic or hypoechoic with thin echogenic border or dysplastic nodules size $>10 \mathrm{~mm}$. Volume redistribution in early stages - liver is enlarged, in advanced stage it's small with relative enlargement of caudate, left lobe or both in comparison to right lobe. ${ }^{33}$ Cirrhosis is the most common cause of portal hypertension.

Finding porto systemic collaterals or portal vein flow reversal may prompt diagnosis of unsuspected portal hypertension and cirrhosis. It is found that the nodular appearance of the liver surface is important in the diagnosis of cirrhosis. ${ }^{32}$ The diagnosis of non-neoplastic parenchymal disease of the liver was made by identification of swollen hepatocytes, vacuolation and decreased cytoplasmic basophilia with disturbance of normal regularity of the liver cells and pronounced anisocytosis of the hepatocytes.

The presence of bile duct fragments, sinusoidal epithelium and connective tissue fibres was an added finding which if present indicates the presence of cirrhosis but the various types of cirrhosis cannot be distinguished by cytological methods. ${ }^{35}$ However for the accurate final diagnosis, FNAC examination is needed, as the tissue type cannot be detected by USG. Guided FNAC is very useful in the diagnosis of hepatic lesions as it is a quick, safe, simple, cost-effective and accurate method.

FNAC can accurately distinguish non-neoplastic from neoplastic lesions, categorize different non-noeplastic lesions and differentiate primary from metastatic tumors. In conclusion we would like to say that USG is a rapid, non-ionizing, non-invasive, safe, relatively reliable, economical investigation and does not require special preparation prior to the examination. By this rapid method, even small lesions with subtle difference in reflectivity can be detected.

It is also very helpful in diagnosing or ruling out any other associated disease or any pathology of gallbladder, kidneys, pancreas, spleen, lymph node or any other organ simulating liver disease. It is highly sensitive in diagnosing focal liver lesions, liver abscess and fatty liver which constituted majority of lesions in the present study.

FNAC offers accuracy without major complications and minimal interventions at low cost. Despite the high diagnostic accuracy, sensitivity and specificity by the USG and FNAC for diffuse liver lesions, the histological diagnosis still remains the gold standard. Despite the minimum drawback, it is evident from this study that USG has a wide applicability in diagnosis of liver lesions. 


\section{REFERENCES:}

1. Beckingham J, Kraig J. Ryde SD. ABC of disease of liver pancreas and biliary tract. BMJ March 2001; 323: 33-36.

2. Christopher J Harvey, James M Pilcher, Robert J Eckersley, Martin JK Bomley, David 0 Cosgrove. Advances in Ultrasound Clin. Radiol. 2002; 57: 157-177.

3. Tchelepi, Hisham MD, Rails W, Philip W. Ultrasound of focal liver masses. Ultrasound Quarterly 2004; 20(4): 155-169.

4. Salani V Duyshant, Saneev D Kalu. Imaging of liver. Oncology. 2004; 9(4): 385-395.

5. Mallikarjuna CM Swamy, CA Arathi, CR Kodandaswamy. Value of ultrasonography guided fine needle aspiration cytology in the investigative sequence of hepatic lesions with an emphasis on hepatocellular carcinoma. J Cytol. 2011 Oct-Dec; 28 (4): 178-184.

6. Sheik T S M, Behbdoni A, Chisti F A, Sheik Z, PR Hira et al. Diagnosis of abdominal hydatid cyst The role of ultrasound and ultrasound guided fine needle aspiration cytology. International Journal of Medical Principles and Practice. 2002; 11(04).

7. Das DK, Bharnbani Chandras, Pant CS. Ultrasound guided FNAC diagnosis of hydatid disease of the abdomen and thorax. Diagn Cytopathol. 1995; 12(2): 173-176.

8. Gupta P, Dhar S, Strickland N H. Fibrolamellar Carcinoma. Eur J Radiol. 1999; 32: 119-123.

9. Samaraturga H, Wright G. Value of FNAC in the diagnosis of discrete hepatic lesions suspicious for malignancy. Aus. N Z J Surg. 1992; 62:540-44.

10. Nasir TA, Banu NA, Hussain M, Begum AA and Ali H. Correlation of ultra-sonographic findings with ultrasound girded FNA of liver lesion. Bangladesh Med Res Counc Bull. 2001; 27(3): 84-9.

11. Ahuja A, Gupta N, Srinivasan R, Kalra N, Chawla Y, Rajwanshi A. Differentiation of Hepatocellular Carcinoma from Metastatic Carcinoma of the Liver - Clinical and Cytological Features. Journal of Cytology.2007; 24 (3): 125-129.

12. Scheible W, Gosink BB, Leopold GR. Gray scale echographic patterns of hepatic metastatic disease. Am J Roentgenol. 1977; 129: 983-987.

13. L. Rubaltelli et al. The role of vascularization in the formation of echogenic patterns of hepatic metastates. BJR.1980; 53: 1166-1168.

14. Rasania A, Pandey CL, Joshi N. Evaluation of FNAC in diagnosis of hepatic lesion. J. Cytol. 2007; 24: 51-4.

15. Alan R. Moody et al. Atypical Hepatic Hemangioma. Radiology, 1993; 188:413-417.

16. Gandolfi L, Leo P, Solmi L, Vitelli E, Verror G, Colecchia A. Natural History of Hepatic Haemangioma Clinical and Ultrasound study. Liver, Biliary, \& Pancreas Gut. 1991; 32: 677-680.

17. Xuh X, Liu GJ, Lum D et al. Characterization of Focal liver lesions using sonography. J. Clin. Ultrasound. 2006; 34: 261-72.

18. Ranson JHC, Lacotio SA, Madayag MA. New diagnostic and therapeutic techniques in the management of pyogenic liver abscess, Am J Roentgenol. 1983; 141: 559-560.

19. Bhambhani S, Kashyap V. Amoebiasis. Diagnosis of aspiration and exfoliative cytology. Cytopathology. 2001; 12: 328-33.

20. Orell S. Gladwyn Leiman. Liver and Spleen -Fine Needle Aspiration Cytology of liver and spleen. Orell S, Daniel Whitetaker, Forrester. 4th Edition, Elsevier Churchill Livingstone. 2005; 10:293308. 
21. Blanco Quintana F, Novella Arribas B, Sanchez Molini P, Sanz Sanz J. Descriptive studies of 39 cases of hepatic abscess of pyogenic and amoebic origin. An Med Intema. October 1995; 12 (10): 477-84.

22. Salvador 5, Jose Maria Salles, Luis Alberto Moraes, Costa Sales M. Hepatic ameobiasis. Braz J Infect Dis. 2003; 7 (2): 11-13.

23. Azhar Jawaid Bukhari, Khali Javed Abid. Amoebic liver abscess-Clinical presentation and diagnostic difficulties. Kuwait Medical Journal. 2003; 35 (3): 183-186.

24. Kuligowska E, Noble J. Sonographic features of hepatic abscess. Semin Ultrasound. 1983; 4: 102116.

25. Gossin KB. Intrahepatic focal liver lesions- Differential diagnosis. AJR. 1981; 137: 763-767.

26. Abdelouafi A, Ousehal A, Vuzidane, Kadiri R. Ultrasonography in the diagnosis of liver abscesses. Ann Radiol. (Paris). March 1993; 36 (4): 286-92.

27. Richard M. Spiegel et al. Ultrasonography of Primary Cysts of the Liver. AJR. 1978 Vol. 131; 235238.

28. Clifford E. Roemer et al. Hepatic Cysts.AJR.1981; 136: 1065 -1070.

29. Jouinis 5, Menif E, Sehilis S, Bensafta Z, Chaemak L, Belaid S et al. Value of ultrasonics in differential diagnosis of hydatid cyst of liver and other solid masses. J Radiol. Aug 1996; 77 (8): 563-9.

30. Lt. Col.R.N. Bagga. Accidental Percutaneous Aspiration of Hydatid cysts. IJRI. 1988; Vol.42: 281283.

31. Hisham Tchelepi, Philip W. Ralls, Randall Radin, Edward Grant. Sonography of Diffuse Liver Disease. 2002; September 1:21: 9.

32. Yang PM, Sheu JC, Huang GT. Ultrasongraphic appearance of the surface changes of the liver- It's accuracy and value in the diagnosis of liver cirrhosis. Chinese J Gastroenterol. 1986; 3: 105-13.

33. Gorg C, Weide R, Schwerk WB. Lymphoma infiltration of the liver- spectrum of ultrasound characteristics in 47 patients. J Gastroenterol.1994; 32: 597-602.

34. Soyer P, Van Beers B, Teillet-Thiebaud F. Hodgkin's and non-Hodgkin's hepatic lymphomasonographic findings. Abdom Imaging 1993; 18: 339-343.

35. Ahmed A. Mohmmed, Shawgi Elsiddig, Mohamed Abdullhamid, Gasim I. Gasim and Ishag Adam. Ultrasound - guided fine needle aspiration cytology and cell block in the diagnosis of focal liver lesions. Khartoum Hospital, Sudan. 2012 September; 7:183-188. 


\begin{tabular}{|c|c|c|c|c|c|c|}
\hline \multirow{2}{*}{$\begin{array}{c}\text { Age } \\
\text { groups } \\
\text { (years) }\end{array}$} & \multicolumn{2}{|c|}{ Male } & \multicolumn{2}{c|}{ Female } & \multicolumn{2}{c|}{ Total } \\
\cline { 2 - 7 } & No. & $\mathbf{0}$ & No & $\mathbf{0}$ & No & $\mathbf{\%}$ \\
\hline Below 10 & 2 & 1.64 & 2 & 1.64 & 4 & 3.36 \\
\hline $11-20$ & 5 & 4.21 & 3 & 2.54 & 8 & 6.74 \\
\hline $21-30$ & 9 & 7.62 & 4 & 3.36 & 13 & 11.01 \\
\hline $31-40$ & 17 & 14.40 & 8 & 6.74 & 25 & 21.19 \\
\hline $41-50$ & 22 & 18.75 & 12 & 10.16 & 34 & 28.81 \\
\hline $51-60$ & 12 & 10.16 & 10 & 8.62 & 22 & 18.73 \\
\hline$>60$ & 6 & 5.08 & 6 & 5.08 & 12 & 10.16 \\
\hline Total & $\mathbf{7 3}$ & $\mathbf{6 1 . 8 6}$ & $\mathbf{4 5}$ & $\mathbf{3 8 . 1 4}$ & $\mathbf{1 1 8}$ & $\mathbf{1 0 0 . 0}$ \\
\hline
\end{tabular}

Table-1: Age \& Sex wise distribution of Focal and diffuse hepatic lesions

\begin{tabular}{|l|c|c|c|c|}
\hline \multirow{2}{*}{$\begin{array}{c}\text { Clinical } \\
\text { symptoms }\end{array}$} & \multicolumn{2}{c|}{ Present } & \multicolumn{2}{c|}{ Absent } \\
\cline { 2 - 5 } & No & \% & No & \% \\
\hline Pain & 53 & 54.08 & 45 & 45.92 \\
\hline Fever & 29 & 29.55 & 69 & 70.45 \\
\hline Hepatomegaly & 31 & 31.63 & 67 & 68.37 \\
\hline Jaundice & 7 & 7.14 & 91 & 92.86 \\
\hline Tenderness' & 19 & 19.38 & 79 & 80.62 \\
\hline
\end{tabular}

Table-2: Distribution of cases based on clinical symptoms of focal liver lesions

\begin{tabular}{|l|c|c|c|c|}
\hline \multicolumn{1}{|c|}{ Lesions } & $\begin{array}{c}\text { Sensitivity } \\
\mathbf{( \% )}\end{array}$ & $\begin{array}{c}\text { Specificity } \\
\mathbf{( \% )}\end{array}$ & $\begin{array}{c}\text { Positive } \\
\text { predictive } \\
\text { value (\%) }\end{array}$ & $\begin{array}{c}\text { Negative } \\
\text { predictive } \\
\text { value (\%) }\end{array}$ \\
\hline PMLT & 84.6 & 93.0 & 81.4 & 94.3 \\
\hline Metastasis & 77.2 & 93.4 & 77.2 & 94.6 \\
\hline Heamangioma & 50 & 97.8 & 60 & 96.7 \\
\hline Liver abscess & 93.9 & 93.8 & 88.5 & 96.8 \\
\hline Cyst & 50.0 & 98.9 & 75.0 & 96.8 \\
\hline Hydatid lesion & 80 & 98.9 & 80.0 & 98.9 \\
\hline \multicolumn{5}{r|}{ Table-3: Statistical values of the focal diseases lesions } \\
\hline
\end{tabular}




\begin{tabular}{|l|c|c|}
\hline \multicolumn{1}{|c|}{ Ultrasound diagnostic } & Number of cases & Percentage \\
\hline Fatty liver & 9 & 45.0 \\
\hline Cirrhosis & 6 & 30.0 \\
\hline Hepatitis & 2 & 10.0 \\
\hline $\begin{array}{l}\text { Diffuse Hepatocellular } \\
\text { carcinoma }\end{array}$ & 3 & 15.0 \\
\hline \multicolumn{1}{|c|}{ Total } & $\mathbf{2 0}$ & $\mathbf{1 0 0 . 0 0}$ \\
\hline
\end{tabular}

Table-4: Distribution of diffuse hepatic lesions by ultrasonography

\begin{tabular}{|l|c|c|}
\hline \multicolumn{1}{|c|}{ FNAC diagnosis } & Number of cases & Percentage \\
\hline Fatty liver & 10 & 50.0 \\
\hline Cirrhosis & 6 & 30.0 \\
\hline Hepatitis & 2 & 10.0 \\
\hline Diffuse hepatocellular carcinoma & 2 & 10.0 \\
\hline Total & $\mathbf{2 0}$ & $\mathbf{1 0 0 . 0 0}$ \\
\hline
\end{tabular}

Table-5: Distribution of diffuse hepatic lesions by FNAC

\begin{tabular}{|l|c|c|c|c|}
\hline & Sensitivity & Specificity & PPV\% & NPV\% \\
\hline Fatty liver & 80.0 & 90.0 & 88.9 & 81.18 \\
\hline Cirrhosis & 83.3 & 92.8 & 83.33 & 92.8 \\
\hline Hepatitis & 50.0 & 94.4 & 50.0 & 94.4 \\
\hline Diffuse HCC & 50.0 & 88.89 & 33.4 & 94.4 \\
\hline \multicolumn{4}{|r|}{ Table-6: Statistical value of the diffuse diseases } \\
\hline
\end{tabular}

\begin{tabular}{|c|c|c|c|c|c|c|c|}
\hline & PMLT & Metastases & $\begin{array}{c}\text { Heaman } \\
\text { gioma }\end{array}$ & $\begin{array}{c}\text { Liver } \\
\text { abscess }\end{array}$ & Cyst & Hydatid & Total \\
\hline PMLT & $\begin{array}{c}22 \\
(84.6 \%)\end{array}$ & $\begin{array}{c}3 \\
(11.6 \%)\end{array}$ & - & $\begin{array}{c}1 \\
(3.8 \%)\end{array}$ & - & - & $\begin{array}{c}26 \\
(100 \%)\end{array}$ \\
\hline Metastases & \begin{tabular}{|c|}
3 \\
$(13.8 \%)$ \\
\end{tabular} & $\begin{array}{c}17 \\
(77.2 \%) \\
\end{array}$ & $\begin{array}{c}1 \\
(4.5 \%) \\
\end{array}$ & $\begin{array}{c}1 \\
(4.5 \%) \\
\end{array}$ & & & $\begin{array}{c}22 \\
(100 \%)\end{array}$ \\
\hline Heamangioma & \begin{tabular}{|c|}
1 \\
$(16.7 \%)$ \\
\end{tabular} & $\begin{array}{c}2 \\
(33.3 \%) \\
\end{array}$ & $\begin{array}{c}3 \\
(50 \%) \\
\end{array}$ & - & - & - & $\begin{array}{c}6 \\
(100 \%) \\
\end{array}$ \\
\hline Liver abscess & \begin{tabular}{c|}
1 \\
$(3.05 \%)$ \\
\end{tabular} & - & $\begin{array}{c}1 \\
(3.05 \%)\end{array}$ & $\begin{array}{c}31 \\
(93.9 \%)\end{array}$ & - & - & $\begin{array}{c}33 \\
(100 \%)\end{array}$ \\
\hline Cyst & - & - & - & $\begin{array}{c}2 \\
(33.3 \%)\end{array}$ & $\begin{array}{c}3 \\
(50 \%)\end{array}$ & $\begin{array}{c}1 \\
(16.07 \%)\end{array}$ & $\begin{array}{c}6 \\
(100 \%)\end{array}$ \\
\hline Hydatid & & & & & $\begin{array}{c}1 \\
(20 \%) \\
\end{array}$ & $\begin{array}{c}4 \\
(80 \%) \\
\end{array}$ & $\begin{array}{c}5 \\
(100 \%) \\
\end{array}$ \\
\hline Total & \begin{tabular}{c|}
27 \\
$(27.6 \%)$
\end{tabular} & $\begin{array}{c}22 \\
(22.05 \%)\end{array}$ & $\begin{array}{c}5 \\
(5.1 \%)\end{array}$ & $\begin{array}{c}35 \\
(35.7 \%)\end{array}$ & $\begin{array}{c}4 \\
(4.0 \%)\end{array}$ & $\begin{array}{c}5 \\
(5.1 \%)\end{array}$ & $\begin{array}{c}98 \\
(100 \%)\end{array}$ \\
\hline
\end{tabular}




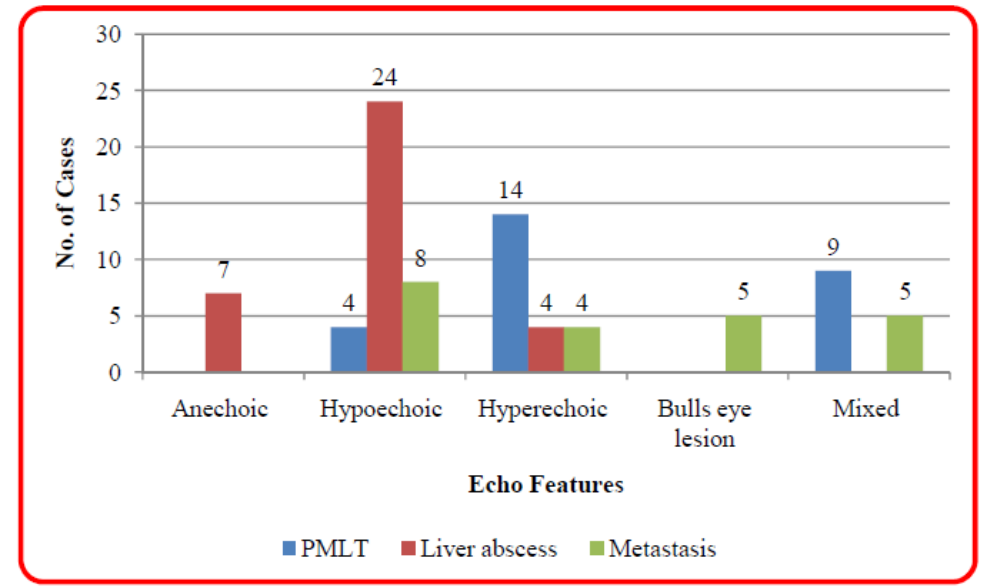

Fig. 1: USG features of focal hepatic lesions

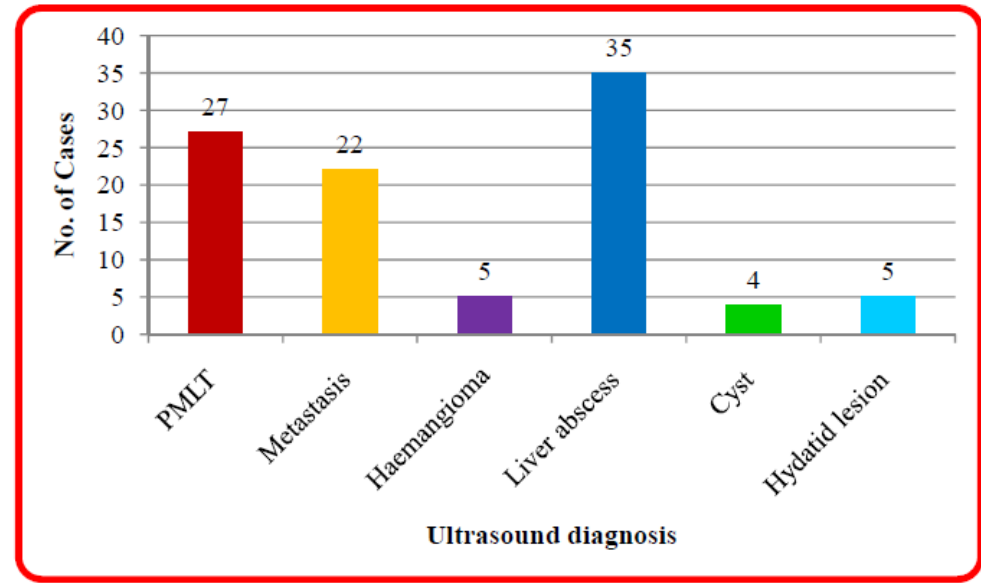

Fig. 2: Distribution all over liver of focal hepatic lesions by USG

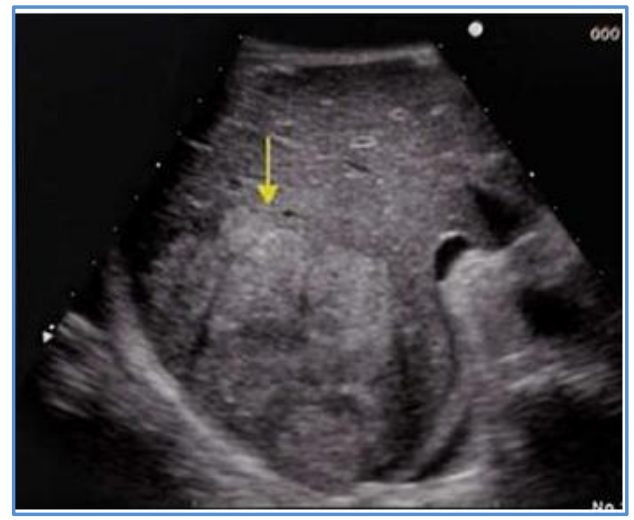

Fig. 3: USG showing large bright echogenic hemangioma liver (Arrow)

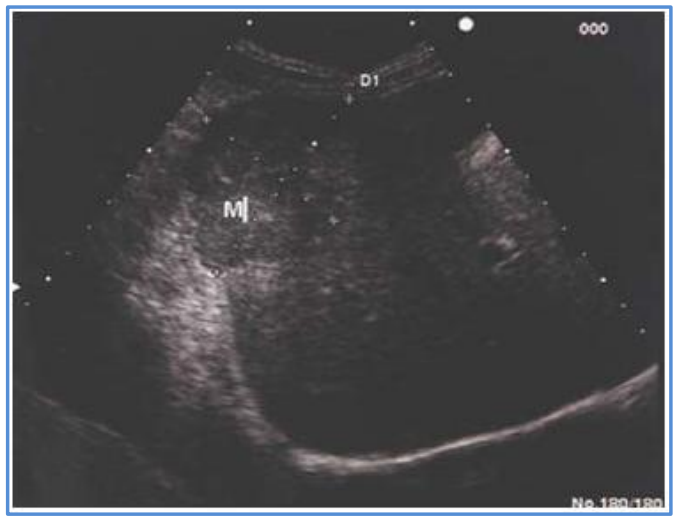

Fig. 4: USG showing irregular solid mass, hepatocellular carcinoma (M) 


\section{REVIEW ARTICLE}

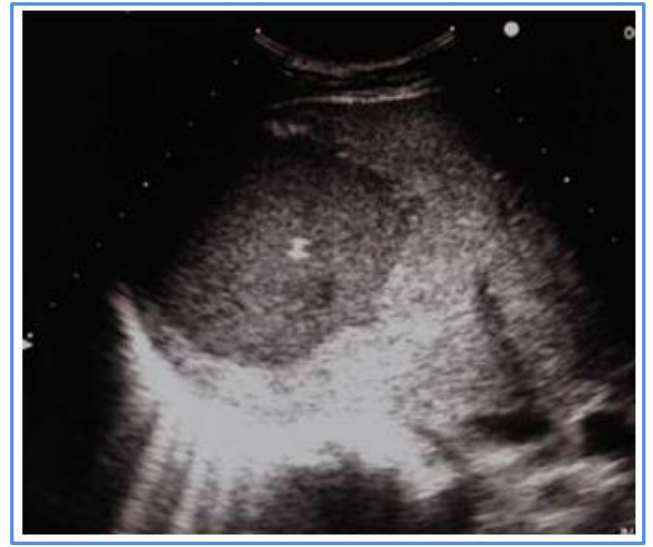

Fig. 5: USG showing large, capsulated with internal echoes - evolved liver abscess

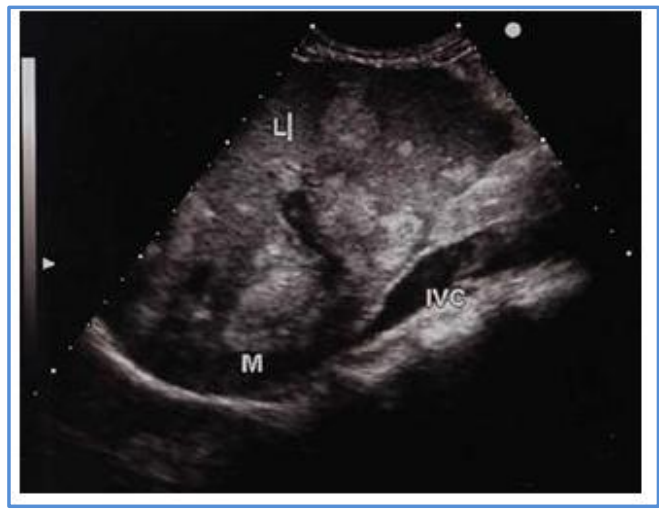

Fig. 7: USG showing multiple liver (L) metastasis $(\mathrm{M})$ with thrombus in inferior vena cava (IVC)

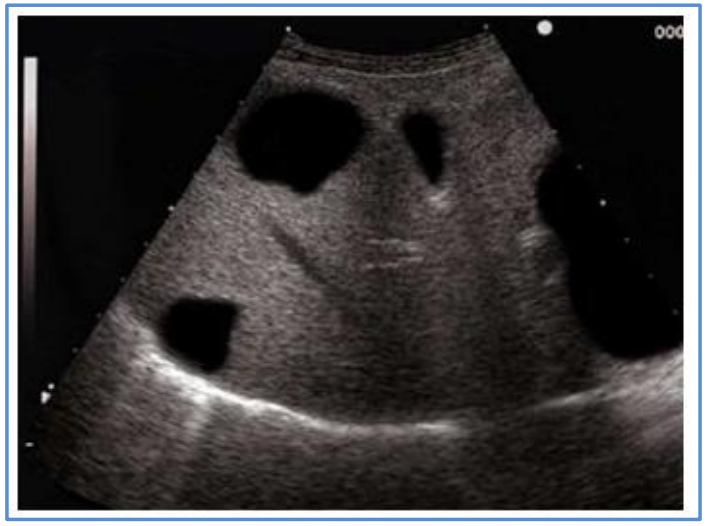

Fig. 9: USG showing multiple anechoic liver cysts of variable sizes

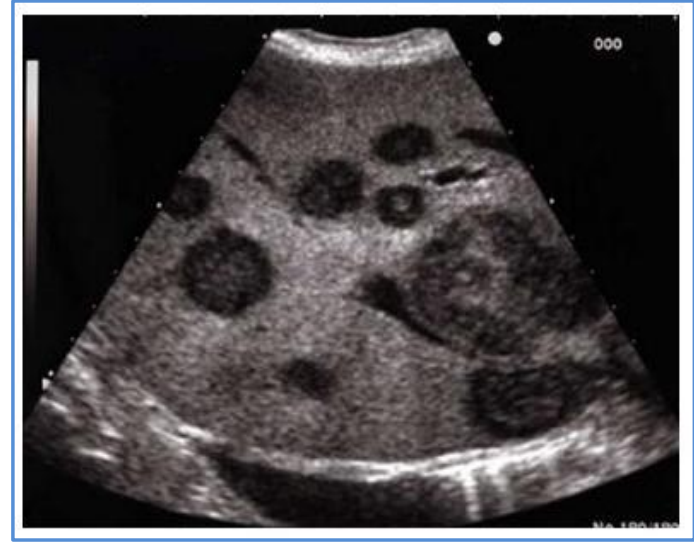

Fig. 6: USG showing multiple, nodules of variable sizes and echoes- liver abscesses

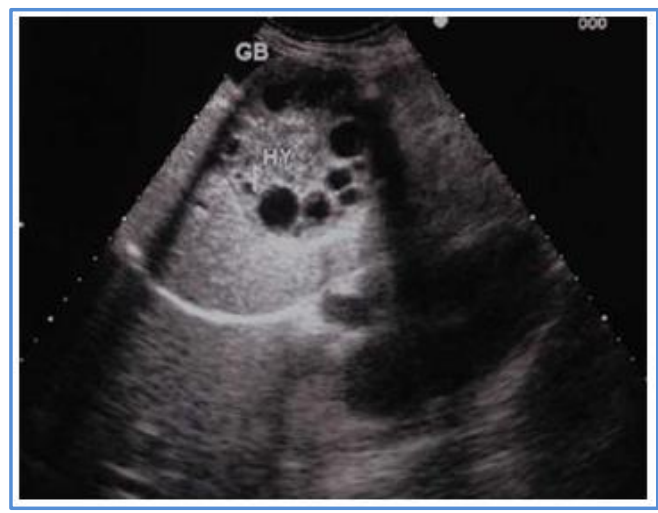

Fig. 8: USG showing large hydatid cyst (hy) with multiple daughter cysts near gallbladder(GB)

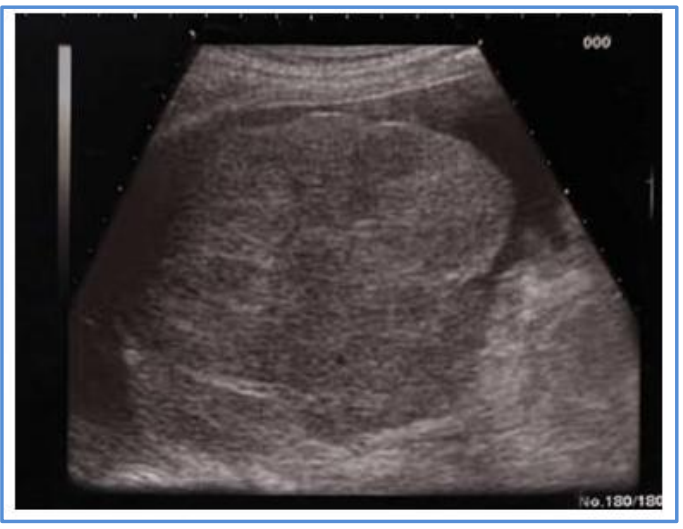

Fig. 10: USG showing bright cirrhotic small irregular outline liver with ascitis 


\section{REVIEW ARTICLE}

\section{AUTHORS:}

1. Hima Bindu P.

2. Anil Kumar Shukla

3. N. Krishnappa

\section{PARTICULARS OF CONTRIBUTORS:}

1. Senior Resident, Department of Radiodiagnosis, Kempegowda Institute of Medical Sciences, K. R. Road, V.V. Puram, Bangalore.

2. Professor, Department of Radiodiagnosis, Kempegowda Institute of Medical Sciences, K. R. Road, V.V. Puram, Bangalore.

3. Professor \& HOD, Department of Radiodiagnosis, Kempegowda Institute of Medical Sciences, K. R. Road, V.V. Puram, Bangalore.

\section{NAME ADDRESS EMAIL ID OF THE CORRESPONDING AUTHOR:}

Dr. Anil Kumar Shukla,

77, Himagiri Apartment, Flat 4C, $15^{\text {th }}$ Cross, $4^{\text {th }}$ Main,

Malleshwaram,

Bangalore-560055, Karnataka.

Email: shookla2007@yahoo.co.in

Date of Submission: 20/08/2014. Date of Peer Review: 21/08/2014. Date of Acceptance: 11/09/2014. Date of Publishing: 18/09/2014. 